\section{Faserforschung und

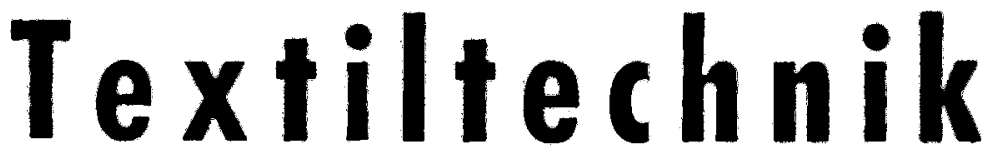

WISSENSCHAFTLICH-TECHNISCHE ZEITSCHRIFT FÜR DIE CHEMIEFASER- UND TEXTILINDUSTRIE

\section{BEGRUNDET VON \\ ERICH CORRENS UND WALTER FRENZEL}

HERAUSGEGEBEM VON ERICH CORRENS

WOLFGANG BOBETH - HANS BOHRINGER - HERMANN KLARE

BURKART PHILIPP - CHRISTIAN RUSCHER

SCHRIFTLEITER J. BRAMER UND I. RUSCHER

\section{AUS DEMINHALT}

Polo-Friz, Rosi und Merani Zusammenhänge zwischen Feinstruktur und Eigenschaften von Polyamid 6 I. Röntgenmessungen an Faserstoffen in Abhängigkeit von der Reckung

Dietrich, Tolsdorf und Reinisch Zur Schmelzviskosität von Polycaprolactam mit niedrigen

Aminoendgruppenkonzentrationen

Čefelín und Markes

Die Polykondensation des Bis-(2-hydroxyäthyl)-terephthalats in dünnen Sch melzeschichten

Schleicher, Linow und Schubert

Untersuchungen zur Quellung und Lösung von Cellulose

in aminhaltigen Flüssigkeitsgemischen 4. Mitt.: Zum Verhalten von Cellulose in ammoniak- und salzhaltigen

Lösungsmittelsystemen

von Hornuff und Heise

Beeinflussung der elektrostatischen Aufladung und der Reibung von Polyamidseide durch Fettsäurepolyglykolester

Kurze Mitteilungen

Winkler, Pietschmann und Götze Zur Bestimmung des Weißgrades an Prüflingen mit durchbrochenem Muster

Winkler, Götze und Pietschmann Zur Bestimmung des Weißgrades an optisch aufgehelltem Prüfgut

Ewert und Dinse

Über die Anwendung der Trübungsmessung zur Titandioxidbestimmung

in mattierten

Polyäthylenterephthalatmaterialien

Neue Büicher

Patentschau

Literaturschau 


\title{
Kybernetische Methoden in der Chemie und chemischen Technologie
}

\author{
W. W. KAFAROW
}

(Übersetzung aus dem Russischen)

Bearbeitet und herausgegeben von Dr.-Ing. KLAUS HARTMANN

in Zusammenarbeit mit einem Übersetzerkollektiv

1971. XII, 483 Seiten - 207 Abbildungen - 53 Tabellen - gr. $8^{\circ}$ - Leinen $44,-M$

Bestell-Nr. 7614560 (5854)

Die Anwendung kybernetischer Methoden und Verfahrensweisen bei der Untersuchung von chemischen Prozessen hat sich als äußerst fruchtbar erwiesen. Durch die konsequente Anwendung der Systemtheorie, mathematischen Modellierung, optimalen Versuchsplanung, Stabilitätsuntersuchungen usw. wird der Entwurf und die Steuerung von chemischen Anlagen auf eine qualitative neue Stufe gehoben. Im vorliegenden Werk erschließt der hervorragende Vertreter und Pionier der „chemischen Kybernetik“, das Korrespondierende Mitglied der Akademie der Wissenschaften der UdSSR, Prof. W. W. Kafarow, alle erforderlichen Quellen, um in das Gesamtgebiet kybernetischer Methoden, die mit Erfolg in der Chemie und chemischen Technologie angewendet wurden, einzudringen.

Ausgehend von einer Einführung in die Grundelemente der Kybernetik werden Systeme, Modelle, Modellierung, Regelung, Steuerung, Information, Digital- und Analogrechner abgehandelt, wobei immer der konkrete chemische Proze $B$ im Vordergrund steht.

Auf diesen Grundlagen aufbauend wird eine umfassende Analyse chemisch-technologischer Prozesse durchgeführt, das methodische Vorgehen bei der mathematischen Modellierung dargestellt und ein Überblick über die wichtigsten Optimierungsverfahren gegeben.

Ein bedeutender Teil des Buches ist der Analyse, Aufstellung und Lösung von Modellen der Typenprozesse (Apparate mit idealer Verdrängung, Apparate mit idealer Vermischung, Diffusionsmodell, kombinierte Reaktormodelle) ohne und mit Berücksichtigung des Wärmetransportes gewidmet.

Dabei werden solche wichtigen Fragen wie die Reaktorstabilität besprochen.

Bestellungen durch eine Buchhandlung erbeten

A K A D E M I E - VERLAG B ERL I N 

DK $677.494 .675^{\prime} 126: 677.021 .123 .3: 677.014 .845: 677.014 .87: 677.016 .673 .45$
677.842 .3

Polo-Friz, Luigi, Rosi, Vizenzo, und Merani, Gianfranco Zusammenhänge zwischen Feinstruktur und Eigenschaften von Polyamid 6

1. Röntgenmessungen an Fasersoffen in Abhängigkeit von der Reckung

Faserforsch. u. Textiltechnik 23 (1972) 8, S. 319-324. 14 Ibb., 24 Lit.

Jis wird cine Methode beschrieben, die es gestattet, mit Hilfe der Röntgenstreıung die strukturellen Veränderungen unterschiedlich kalt- und warmgereckter Polyamid-6-Faserstoffe zu untersuchen. Die Messungen wurden einerseits an Faserstoffen mit konstanter Ausgangsfeinheit und demgemäl unterschiedlichen Feinheiten des gereckten Materials, andererseits an Faserstoffen, bei denen durch Einsatz unterschiedlicher Ausgang

Yur liestimmung besagter Zusammenhänge wird die Intensität der Meridian(diatropen)-Reflexe (002) bei etwa $2 \Theta=11^{\circ}$ bzw. (004) bei etwa $2 \Theta=21^{\circ}$ (diatropen)-Reflexe $(002)$ bei etwa $2 \Theta=11^{\circ}$ bzw. $(004)$ bei etwa $2 \Theta=21^{\circ}$ unter besonderen Bedingungen gemessen. Anschließend wird die gleiche schiedlichen Tomperaturen festzustellen.

])K $678.675^{\prime} 126-498.4: 532.135: 532.133: 541.24$

Dielrich, Klaus, Tolsdorf, Siegfried, und Reinisch, Gerhard Zur Sehmelzviskosität von Polycaprolactamen mit niedrigen Aminoendgruppenkonzentrationen

Faserforsch. u. Textiltechnik 23 (1972) 8, S. 325-328. 3 Abb., 6 Tab., 12 Lit.

An Polycaprolactamen mit sehr weitgehend cingeschränkter Nachpolykondensationsfähigkeit wurden bei 230 bis $295^{\circ} \mathrm{C}$ rotationsviskosimetrische Messungen durchgefuhrt. Die fü $250^{\circ} \mathrm{C} / 10^{2} \mathrm{~s}^{-1}$ ermittelte Beziehung zwischen der Schmelzviskosität der zu 75 bis $40 \%$ acylierten Proben und ihren $\bar{M}_{n}$ wird mit Literaturwerten verglichen.

DK $678.674^{\prime} 524^{\prime} 420: 66.095 .3: 66-914.3: 541.128: 541.127 .1: 541.24$

Čefelin, Pavel, und Markes, liudolf

Die Polykondensation des Bis-(2-hydroxyäthyl)-lercphthalats in dünnen Schmelzesschichten

Faserforsch. u. Textiltechnik 23 (1972) 8, S.329-334. 8 Abb., 2 Tab., 18 Lit.

Für Monomerenansätze unter $1 \mathrm{~g}$ wurden Polykondensationsverfahren in statischen und rotierenden, dünnen Schmelzeschichten ausgearbeitet, in denen Masseänderungen des durch Destillation eintrotenden Verlustes an Monomeren sowie der Einfluß von Katalysatorkonzentration, Polymeri sationsgrad und Diffusion auf die Reaktionsgesehwindigkeitskonstanten der Polykondensation untersucht werden können. Die vorgeschlagenen Verfahren eignen sich im besonderen zur qualitativen Bewertung der Ausgangs-
produkte im Hinblick auf thre Eignung für die Polyesterherstellung.

DK $661.728: 547.458 .81: 66.062 .85: 532.73: 532.77: 532.135$

Schleicher, Harry, Linow, Karl-Joachim, und Schubert, Klaus Untersuchungen zur Quellung und Lösung von Cellulose in aminhaltigen Flüssigkeitsgemischen

4. Mitt.: Zum Verhalten von Cellulose in ammoniak- und salzhaltigen Lösungsmittelsystemen

Faserforsch. u. Textiltechnik 23 (1972) 8, S. 335-340. 3 Abb., 4 Tab. 12 Lit.

Die Auflösung von Cellulose in Lösungen von Ammoniak und Natriumsalzen in Formamid, Dimethylsulfoxid, Morpholin oder A thanolamin wird beschriesulfoxid/äthanolamin/Ammoniak/Natriumbromid sowie an verdünnten und konzentrierten Celluloselösungen werden mitgeteilt und Rückschlüsse auf den Lösungszustand der Cellulose abgeleitet.
DK $677.494 .675^{\prime} 126-486.2: 537.221: 677.862 .517 .1 ; 547.29^{\prime} 26: 541.183$ von Hornuff, Günther, und Ileise, Martin

Beeintlussung der clektrostatisehen Aufladung und der Reibung von Polyamidseide durch Fetsäurepolyglykolester Faserforsch. u. Textiltechnik 29 (1972) 8, S. 340-346. 11 Abb., 24 Lit.

Alsgehend von Vntersuchungen über die grenzflächenaktiven Eigenschaften und die Struktur von Fettsäurepolyglykolestern wird der Linfluß dieser Substanzen auf dic Oberlacheneigensehaten von Polyamidseide crmittelt. Die Wirkung der Fettsäurepolyglykolester als Präparationskomponenten wird durch Widerstands-, Aufladungs- und Reibungsmessungen an präparierter Polyamidseide untersucht

\section{Kurze Milteilungen}

DK $677.65: 535.668 .6: 535.65: 535.31$ 는

Winkler, Friedrich, Pietschmann, Sieglinde und Götze, Wolfgang

Zur Bestimmung des Weißgrades an Prüflingen mit durchbrochenem Muster

Faserforsch. u. Textiltechnik 23 (1972) 8, S. 347.1 Abb., $1 \mathrm{Tab}$

DK $677.1 / .5: 677.044 .314 .5: 677.016 .6: 677.827 .3: 535.668 .6$

Winkler, Friedrich, Götze, Wolfgang, und Pietschmann, Sieglinde

Zur Bestimmung des Weißgrades an optisch aufgehelltem Prüfgut

Faserforsch. u. Textiltechnik 23 (1972) 8, S. 348-349. 3 Abb., $1 \mathrm{Tab}$.

DK $678.674^{\prime} 524^{\prime} 420: 678.047 .6: 546.824-31: 543.436$

Ewert, Karl-Ileinz, und Dinse, Hans-Dieter

Über die Anwendung der Trübungsmessung zur Titandioxidbestimmung in matierten Polyäthylenterephthalatmaterialien

Faserforsch. u. Textiltechnik 28 (1972) 8, S. 349-350. 2 Abb., 8 Lit.

Neue Bücher S. 351-352.

Patentschau S. 352-355.

Literaturschau S. 355-358. 


\section{Kunstharz - lonenaustauscher}

\section{Plenar- und Diskussionsvorträge des Symposiums im Juni 1968 in Leipzig}

(lonenaustauscher in Einzeldarstellungen)

1970. X, 702 Seiten - 447 Abbildungen - 102 Tabellen - gr. $8^{\circ}$ - Leinen 120, - M;

Bestell-Nr. $7613453(2120 / 8)$

Diese Veröffentlichung ist dem Symposium gewidmet, das sich mit der Synthese, Theorie und Anwendung der Kunstharzionenaustauscher, die vor 30 Jahren erstmals angewendet wurden, beschäftigt.

Die einzelnen Beiträge dieses Berichtes beschäftigen sich mit inogenen funktionellen Gruppen und von ihnen abgeleitete Materialien.

Bei dieser Darlegung steht die industrielle Herstellung naturgemäß sehr stark im Vordergrund. Die Bedeutung dieser modernen Hilfsmittel der Forschung und Industrie - in dem recht kurzen Zeitraum seit ihrer Entwicklung - wird für die wichtigsten Gebiete der lonenaustauscheranwendung dargelegt.

Bestellungen durch eine Buchhandlung erbeten

A K A D E M I E - VER LAG. B E R L I N

Die Zeitschrift „Faserforschung und Textiltcchnik“ erscheint monatlich in Heften zu 48 Textseiten im Format A 4. Der Preis für das Einzelheft beträgt M 15, - (Sonderpreis für DDR M 9,-), für den Vierteljahresbezug M1 45,- (Sonderpreis für DDR M 27, -), zuzúgl. jiestellgeld. Die Bereehnung erfolgt zu Beginn eines Vierteljahrs für 3 Hefte. Bestellungen aus dem (iel,iet der Ientschen Demokratischen Republik an ein Postamt, eine Bbuchhandlung oder den Verlag, aus der Deutschen Bundesrepublik an eine Buchhandlung oder dic Auslicferungsstelle Kunst und Wissen, Erieh Bicber, 7 Stuttgart 1, Wilhelmstraße 4-6, aus dem Ausland an eine Importbuchhandlung, den Deutschen Buch-Export und -Import GmbH., 701 Leipzig, Postsehließfach 276, oder den

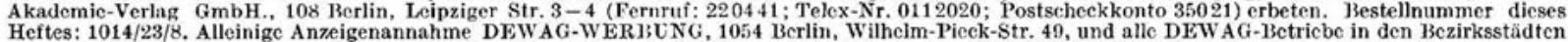

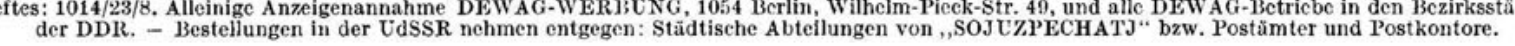

Herausgeber und verantwortlich für den Inhalt: Prof. Dr. Dr. Erich Correns, Institut für Polymerenchemie der Deutschen Akademie der Wissenschaften zu Berlin, 153 Teltow-Scehof, Fernruf: Teltow 4831; Prof. Dr.-Ing. halil. Wolfgang Bobeth, Institut für Technologie der Fasern der Deutschen Akademie der Wissenschaften zu J3erlin, 801 Dresden, Hohe Str. 6, Fernruf: 44721; Prof.-Dr. Ing. Hans Böhringer. Institut für Textiltechnologie der Chemiefasern Rudolstadt, Fernruf: Rudolstadt 2031; Prof. Dr. Hermann Klare, Prof. Dr. habil. Jurkart Philipp und Dr. habil. Christian Ruscher, Institut fur Polymerenchemie der Deutschen A kademie der Wissenschaften zu Berlin, 153 Teltow-Scehof, Fernruf: Teltow 4831. Sehriftleiter: Joachim 13rümer und Dipl.-Chem. Ingeborg Ruscher, 153 Teltow-Seehof, Kantstr. 55. Verlag: Akademic-Verlag GmbH., 108 Berlin, Leipziger Str. 3-4, Satz und Druck: Druckhaus, Maxim Gorki", 74 Altenburg. - Veröffentlicht unter der Lizenznummer 1280 des Presseamtes beim Vorsitzenden des Ministerrates der Deutschen Demokratischen Republik.

Manuskriptsendungen sind an einen der Herausgeber oder die Schriftleitung zu richten. Für Inhalt und Form gelten die ,,Richtlinien für die Annahme und Abfassung von Beiträgen“, erhältlich von der Schriftleitung. Die Verfasser größerer wissenschaftlicher Arbeiten erhalten außer dem Honorar ein Heft und 50 Sonderdrucke ihrer Arbeit unentgeltich. Nachdrucke sowie t'bersetzungen in fremde Sprachen des Inhalts dieser \%eitschrift und deren Verbreitung - auch auszugsweise mit Quellenangabe - bedürien der schriftlichen Vereinbarung mit dem Verlag. 